\title{
FINITE DIMENSIONAL QUASI-HOPF ALGEBRAS WITH RADICAL OF CODIMENSION 2
}

\author{
Pavel Etingof and Shlomo Gelaki
}

\section{Introduction}

This paper is motivated by the problem of classification of finite tensor categories $\mathcal{C}$ over $\mathbb{C}$. The most natural family of finite tensor categories to start with is the one that consists of those $\mathcal{C}$ in which the Frobenius-Perron dimensions of objects are integers. This is, in a sense, the easiest case, since by a result of [EO], such a category is the representation category of a finite dimensional quasi-Hopf algebra. One is thus led naturally to the problem of classifying finite-dimensional quasi-Hopf algebras over $\mathbb{C}$. Like for Hopf algebras, the easiest nonsemisimple finite dimensional quasi-Hopf algebras to understand are those which are basic (i.e., all irreducible representations are 1-dimensional). However, the problem of classifying finite dimensional basic quasi-Hopf algebras is still quite complicated, and the objective of this paper is to do so in the simplest nontrivial case, when the quasi-Hopf algebra has two one-dimensional irreducible representations.

Let us now describe the composition of the paper in more detail.

It is shown in [EO], Proposition 2.17, that a finite dimensional quasi-Hopf algebra over $\mathbb{C}$ with radical of codimension 1 is semisimple and 1-dimensional. On the other hand, there exist quasi-Hopf (in fact, Hopf) algebras, whose radical has codimension 2. Namely, it is known [N] that these are exactly the Nichols Hopf algebras $H_{2^{n}}$ of dimension $2^{n}, n \geq 1$ (one for each value of $n$ ). All these Hopf algebras are basic.

The main result of this paper is that if $H$ is a finite dimensional quasi-Hopf algebra over $\mathbb{C}$ with radical of codimension 2 , then $H$ is twist equivalent to a Nichols Hopf algebra $H_{2^{n}}, n \geq 1$, or to a lifting of one of the four special quasiHopf algebras $H(2), H_{+}(8), H_{-}(8), H(32)$ of dimensions $2,8,8$, and 32 , defined in Section 3. As a corollary we obtain that any finite tensor category which has two invertible objects and no other simple object is equivalent to $\operatorname{Rep}\left(\mathrm{H}_{2^{n}}\right)$ for a unique $n \geq 1$, or to a deformation of the representation category of $H(2)$, $H_{+}(8), H_{-}(8)$, or $H(32)$. In the case of $H(2)$ such a lifting (deformation) must clearly be trivial; for the other three cases we plan to study possible liftings in a later publication.

As another corollary we prove that any nonsemisimple quasi-Hopf algebra of dimension 4 is twist equivalent to $H_{4}$.

Received October 16, 2003. 
Thus, this paper should be regarded as a beginning of the structure theory of finite dimensional basic quasi-Hopf algebras. It is our expectation that this theory will be a nontrivial generalization of the deep and beautiful theory of finite dimensional basic (or, equivalently, pointed) Hopf algebras (see [AS] and references therein).

\section{Preliminaries}

All constructions in this paper are done over the field of complex numbers $\mathbb{C}$.

2.1. Recall ([D]) that a quasi-Hopf algebra is an algebra $H$ equipped with a comultiplication $\Delta$ which is associative up to conjugation by an invertible element $\Phi \in H^{\otimes 3}$ (called the associator), a counit $\varepsilon$, and an antipode $S$ together with two distinguished elements $\alpha, \beta \in H$, satisfying certain axioms. An invertible element $J=\sum_{i} f_{i} \otimes g_{i} \in H \otimes H$ satisfying $(\varepsilon \otimes \mathrm{id})(J)=(\mathrm{id} \otimes \varepsilon)(J)=1$, with inverse $J^{-1}=\sum_{i} \overline{f_{i}} \otimes \overline{g_{i}}$, is called a twist for $H$. Using a twist $J$ for $H$, one can define a new quasi-Hopf algebra structure $H^{J}=\left(H, \Delta^{J}, \varepsilon, \Phi^{J}, S^{J}, \beta^{J} \alpha^{J}, 1\right)$ on the algebra $H$, where

$$
\begin{gathered}
\Delta^{J}(h)=J \Delta(h) J^{-1}, h \in H, \\
\Phi^{J}:=(1 \otimes J)(\mathrm{id} \otimes \Delta)(J) \Phi(\Delta \otimes \mathrm{id})\left(J^{-1}\right)(J \otimes 1)^{-1}, \\
\alpha^{J}:=\sum_{i} S\left(\overline{f_{i}}\right) \alpha \overline{g_{i}}, \beta^{J}:=\sum_{i} f_{i} \beta S\left(g_{i}\right)
\end{gathered}
$$

and

$$
S^{J}(h):=\beta^{J} S(h)\left(\beta^{J}\right)^{-1}, h \in H .
$$

We say that two quasi-Hopf algebras $H$ and $K$ are twist equivalent if $K$ and $H^{J}$ are isomorphic as quasi-Hopf algebras for some twist $J$ for $H$. In this situation $K$ and $H$ are categorically indistinguishable in the sense that $\operatorname{Rep}(H)$ and $\operatorname{Rep}(K)$ are tensor equivalent. If $H$ is twist equivalent to a Hopf algebra, we say that the associator of $H$ is trivial. We refer the reader to [D] for more details.

Recall that a finite rigid tensor category $\mathcal{C}$ over $\mathbb{C}$ is a rigid tensor category over $\mathbb{C}$ with finitely many simple objects and enough projectives such that the neutral object 1 is simple (see [EO]). Let $\operatorname{Irr}(\mathcal{C})$ denote the (finite) set of isomorphism classes of simple objects of $\mathcal{C}$. Then to each object $X \in \mathcal{C}$ there is attached a positive number FPdim $(X)$, called the Frobenius-Perron (FP) dimension of $X$ (namely it is the largest positive eigenvalue of the operator of multiplication by $X$ in the Grothendieck ring of $\mathcal{C}$ ), and the Frobenius-Perron (FP) dimension of $\mathcal{C}$ is defined by

$$
\operatorname{FPdim}(\mathcal{C})=\sum_{X \in \operatorname{Irr}(\mathcal{C})} \operatorname{FPdim}(X) \operatorname{FPdim}\left(P_{X}\right)
$$

where $P_{X}$ denotes the projective cover of $X[\mathrm{E}]$. For example, if $\mathcal{C}$ is equivalent to $\operatorname{Rep}(H), H$ a finite dimensional quasi-Hopf algebra, then $\operatorname{FPdim}(X)=\operatorname{dim}(X)$ 
and $\mathrm{FP} \operatorname{dim}(\mathcal{C})=\operatorname{dim}(H)$; in particular the FP-dimensions are integers. Moreover, by Proposition 2.6 in $[\mathrm{EO}], \mathcal{C}$ is equivalent to $\operatorname{Rep}(H), H$ a finite dimensional quasi-Hopf algebra, if and only if the FP-dimensions of its objects are integers.

2.2. Recall that Nichols' Hopf algebra $H_{2^{n}}(n \geq 1)$ is the $2^{n}$-dimensional Hopf algebra generated by $g, x_{1}, \ldots, x_{n-1}$ with relations $g^{2}=1, x_{i}^{2}=0(1 \leq$ $i \leq n-1), x_{i} x_{j}=-x_{j} x_{i}(1 \leq i \neq j \leq n-1)$ and $g x_{i}=-x_{i} g$, where $g$ is a grouplike element and $\Delta\left(x_{i}\right)=x_{i} \otimes 1+g \otimes x_{i}[\mathrm{~N}]$. The Hopf algebra $H_{4}$ is known as the Sweedler's Hopf algebra [S]. Nichols' Hopf algebras are the unique (up to isomorphism) Hopf algebras with radical of codimension 2. It is well known (and easy to check) that $H_{2^{n}}$ is self dual.

Remark 2.1. "Nichols' Hopf algebras" should not be confused with "Nichols' algebras" which are different objects.

2.3. The following is the simplest (and well known) example of a quasi-Hopf algebra not twist equivalent to a Hopf algebra. The 2-dimensional quasi-Hopf algebra $H(2)$ is generated by a grouplike element $g$ such that $g^{2}=1$, with associator $\Phi=1-2 p_{-} \otimes p_{-} \otimes p_{-}\left(\right.$where $\left.p_{+}:=(1+g) / 2, p_{-}:=(1-g) / 2\right)$, distinguished elements $\alpha=g, \beta=1$, and antipode $S(g)=g$. It is not twist equivalent to a Hopf algebra, and any 2-dimensional quasi-Hopf algebra is twist equivalent either to $\mathbb{C}\left[\mathbb{Z}_{2}\right]$ or to $H(2)$. This follows from the fact that tensor category structures on $\operatorname{Rep}\left(\mathbb{C}\left[\mathbb{Z}_{2}\right]\right.$ ) (with usual tensor multiplication) are classified by the group $H^{3}\left(\mathbb{Z}_{2}, \mathbb{C}^{*}\right)=\mathbb{Z}_{2}$ (see e.g. [CE], examples $1.6,1.7$ ).

2.4. Let $H$ be a finite dimensional algebra, and let $I:=\operatorname{Rad}(H)$ be the Jacobson radical of $H$. Then we have a filtration on $H$ by powers of $I$. So one can consider the associated graded algebra $\operatorname{gr}(H)=\bigoplus_{k \geq 0} H[k], H[k]:=I^{k} / I^{k+1}$ $\left(I^{0}:=H\right)$.

The following lemma is standard.

Lemma 2.2. (i) Let $L$ be any linear complement of $I^{2}$ in $H$. Then $L$ generates $H$ as an algebra.

(ii) $\operatorname{gr}(H)$ is generated by $H[0]$ and $H[1]$.

Proof. (i) Let $B \subseteq H$ be the subalgebra generated by $L$. We will show by induction in $k$ that $B+I^{k}=H$ for all $k$. This implies the statement, since $I^{N}=0$ for some $N$. The base of induction, $k=2$, is clear. So assume that $k>2$. By the induction assumption, all we need to show is that $I^{k-1} \subseteq B+I^{k}$. So let us take $a \in I^{k-1}$, and show that $a \in B+I^{k}$. We may assume that $a=i_{1} \cdots i_{k-1}$, where $i_{m} \in I$ for all $m$. Let $\bar{i}_{m} \in L \cap I$ be the unique elements such that $\bar{i}_{m}-i_{m} \in I^{2}$. Then $\bar{a}:=\bar{i}_{1} \cdots \bar{i}_{k-1} \in B$, and $a-\bar{a} \in I^{k}$. We are done.

(ii) Clearly, $\operatorname{gr}(I)$ is the radical of $\operatorname{gr}(H)$. Thus we only need to show that $\operatorname{gr}\left(I^{2}\right)=\operatorname{gr}(I)^{2}$ (then everything follows from (i)). The inclusion $\operatorname{gr}\left(I^{2}\right) \supseteq \operatorname{gr}(I)^{2}$ is clear, so let us prove the opposite inclusion. Since both spaces are graded, it suffices to pick a homogeneous element $a \in \operatorname{gr}\left(I^{2}\right)$ and show that it lies in 
$\operatorname{gr}(I)^{2}$. Suppose $a$ has degree $k$, i.e. $a \in I^{k} / I^{k+1}$. Let $\tilde{a}$ be a lifting of $a$ to $I^{k}$. Then there exist elements $i_{1 j}, \ldots, i_{k j} \in I, j=1, \ldots, m$, such that $\tilde{a}=$ $\sum_{j} i_{1 j} \cdots i_{k j}$. Let $\bar{i}_{1 j}, \ldots, \bar{i}_{k j}$ be the projections of $i_{1 j}, \ldots, i_{k j}$ to $I / I^{2} \subseteq \operatorname{gr}(I)$. Then $a=\sum_{j} \bar{i}_{1 j} \cdots \bar{i}_{k j}$. Since $k \geq 2$, we are done.

2.5. Let $H$ be a basic quasi-Hopf algebra (i.e., all irreducible $H$-modules are 1-dimensional) and let $I:=\operatorname{Rad}(H)$ be the radical of $H$. Clearly the tensor product of any two irreducible representations of $H$ is irreducible and the dual of an irreducible representation is irreducible. This means that $I$ is a quasi-Hopf ideal (i.e. $\Delta(I) \subseteq H \otimes I+I \otimes H, S(I)=I$ and $\varepsilon(I)=0$ ), so we have a quasiHopf algebra filtration on $H$ by powers of $I$. The associated graded algebra $\operatorname{gr}(H)=\bigoplus_{k} H[k]$ is thus also a quasi-Hopf algebra: the operations of $\operatorname{gr}(H)$ are simply the graded (or "degree zero") parts of the operations in $H$. In particular, by definition, the associator of $\operatorname{gr}(H)$ lives in $H[0]^{\otimes 3}$.

We have $H[0]=\operatorname{Fun}(G)$, where $G$ is a finite group (the group of characters of $H)$. Thus the associator of $\operatorname{gr}(H)$ corresponds to an element of $H^{3}\left(G, \mathbb{C}^{*}\right.$ ) (see e.g. [CE],[ENO]). It is also easy to see that $H[1]=\bigoplus_{a, b \in G} \operatorname{Ext}^{1}(a, b)^{*}$.

\section{The main results}

Our first main theorem is the following.

Theorem 3.1. Let $H$ be a finite dimensional quasi-Hopf algebra over $\mathbb{C}$ with radical of codimension 2. Suppose that the associator of $H$ is trivial on 1-dimensional $H$-modules (i.e., the subcategory of Rep $(H)$ generated by 1-dimensional objects is equivalent to $\operatorname{Rep}\left(\mathbb{Z}_{2}\right)$ with the trivial associator). Then $H$ is twist equivalent to a Nichols Hopf algebra $H_{2^{n}}, n \geq 1$.

The proof of the theorem is given in Section 4.

The assumption on the triviality of the associator of $H$ on 1-dimensional modules is clearly essential already for $\operatorname{dim}(H)=2$, because of the existence of the quasi-Hopf algebra $H(2)$. However, this assumption is also essential in higher dimensions, as the following propositions show.

Proposition 3.2. There exist two 8-dimensional quasi-Hopf algebras $H_{ \pm}(8)$ (permuted by the action of the Galois group), which have the following structure. As algebras $H_{ \pm}(8)$ are generated by $g, x$ with the relations $g x=-x g, g^{2}=1$, $x^{4}=0$. The element $g$ is grouplike, while the coproduct of $x$ is given by the formula

$$
\Delta(x)=x \otimes\left(p_{+} \pm i p_{-}\right)+1 \otimes p_{+} x+g \otimes p_{-} x,
$$

where $p_{+}:=(1+g) / 2, p_{-}:=(1-g) / 2$. The associator is $\Phi=1-2 p_{-} \otimes p_{-} \otimes p_{-}$, the distinguished elements are $\alpha=g, \beta=1$, and the antipode is $S(g)=g$, $S(x)=-x\left(p_{+} \pm i p_{-}\right)$.

Proposition 3.3. There exists a 32- dimensional quasi-Hopf algebra H(32), which has the following structure. As an algebra $H(32)$ is generated by $g, x, y$ 
with the relations $g x=-x g, g y=-y g, g^{2}=1, x^{4}=0, y^{4}=0, x y+i y x=0$. The element $g$ is grouplike, while the coproducts of $x, y$ are given by the formulas

$$
\begin{gathered}
\Delta(x)=x \otimes\left(p_{+}+i p_{-}\right)+1 \otimes p_{+} x+g \otimes p_{-} x, \\
\Delta(y)=y \otimes\left(p_{+}-i p_{-}\right)+1 \otimes p_{+} y+g \otimes p_{-} y,
\end{gathered}
$$

where $p_{+}:=(1+g) / 2, p_{-}:=(1-g) / 2$. The associator is $\Phi=1-2 p_{-} \otimes p_{-} \otimes p_{-}$, the distinguished elements are $\alpha=g, \beta=1$, and the antipode is $S(g)=g$, $S(x)=-x\left(p_{+}+i p_{-}\right), S(y)=-y\left(p_{+}-i p_{-}\right)$. Thus, H(32) is generated by its quasi-Hopf subalgebras $H_{+}(8)$ and $H_{-}(8)$ generated by $g, x$ and $g, y$, respectively.

Propositions 3.2 and 3.3 are proved in Section 5.

Nonetheless, it turns out that outside of dimensions 2, 8 and 32 the assumption of the triviality of the associator of $H$ on 1-dimensional modules is satisfied automatically. This striking fact follows from our second main theorem, which is the following.

Theorem 3.4. Let $H$ be a finite dimensional quasi-Hopf algebra over $\mathbb{C}$ with radical of codimension 2. Suppose that the associator of $H$ is nontrivial on 1 -dimensional $H$-modules (i.e., the subcategory of Rep $(H)$ generated by 1 -dimensional objects is equivalent to $\operatorname{Rep}(H(2)))$. Then $\operatorname{gr}(H)$ is twist equivalent to $H(2), H_{+}(8), H_{-}(8)$, or $H(32)$; in particular, the dimension of $H$ is 2,8 or 32 . The quasi-Hopf algebras $H_{+}(8)$ and $H_{-}(8)$ are not twist equivalent.

Theorem 3.4 is proved in Section 6.

Corollary 3.5. Any finite tensor category which has two invertible objects and no other simple object is tensor equivalent to $\operatorname{Rep}\left(H_{2^{n}}\right)$ for a unique $n \geq 1$, or to a deformation of the representation category of $H(2), H_{+}(8), H_{-}(8)$, or $H(32)$.

Proof. Since each simple object is invertible its FP-dimension equals 1 . Hence the $\mathrm{FP}$-dimension of any object in $\mathcal{C}$ is an integer. Indeed, such an object has a filtration for which the successive quotients are simple objects, so the dimension of this object is the sum of the dimensions of the successive quotients, which are 1.

Therefore, by Proposition 2.6 in $[\mathrm{EO}], \mathcal{C}$ is equivalent to a representation category of a finite dimensional quasi-Hopf algebra with radical of codimension 2. The result follows now from Theorems 3.1 and 3.4.

Corollary 3.6. Any nonsemisimple quasi-Hopf algebra of dimension 4 is twist equivalent to $\mathrm{H}_{4}$.

Proof. By dimension counting it follows that $H$ is basic; that is, all irreducible representations of $H$ are 1 -dimensional. Moreover, by Theorem 2.17 in [EO], $H$ has more than one irreducible representation. Let $\chi$ be a non-trivial $1-$ dimensional representation of $H$, and let $P_{\chi}$ denote the projective cover of $\chi$. Then $P_{\chi}=\chi \otimes P_{\varepsilon}$, where $P_{\varepsilon}$ is the projective cover of the trivial representation $\varepsilon$. Therefore 4 equals $\operatorname{dim}\left(P_{\varepsilon}\right)$ times the number of simple objects in $\operatorname{Rep}(H)$. Since both numbers are greater than 1 , we have that they are equal to 2 . So $H$ 
has 2 irreducible representations $\varepsilon$ and $\chi$, with $\chi^{2}=\varepsilon$. The result follows now from Theorems 3.1 and 3.4.

Corollary 3.7. A nonsemisimple finite tensor category $\mathcal{C}$ of FP-dimension 4 is tensor equivalent to $\operatorname{Rep}\left(\mathrm{H}_{4}\right)$.

Proof. It suffices to show that $\mathcal{C}$ has integer FP-dimensions of objects; in this case by Proposition 2.6 in [EO], $\mathcal{C}$ is a representation category of a 4-dimensional quasi-Hopf algebra, and Corollary 3.6 applies.

Let $X$ be a simple object of $\mathcal{C}$ of FP-dimension $d$. If $X \otimes X^{*} \neq 1$ then $X \otimes X^{*}$ contains as constituents 1 and another object. So if $d \neq 1$ then $d \geq \sqrt{2}$. On the other hand, the projective cover $P_{\mathbf{1}}$ of the neutral object has to involve other objects, so it is at least 2-dimensional. This shows that the only chance for $\mathcal{C}$ to have dimension 4 is when all simple objects are 1-dimensional. We are done.

Remark 3.8. We note that semisimple finite tensor categories of FP-dimension 4 are easy to classify. Such a category is either the category of modules over the group algebra of a group of order 4 with associativity defined by a 3-cocycle on this group, or a Tambara-Yamagami category [TY] corresponding to the Ising model (see [ENO], Proposition 8.32).

\section{Proof of Theorem 3.1}

By assumption, the associator of $\operatorname{gr}(H)$ is trivial. Therefore $\operatorname{gr}(H)$ is twist equivalent to a Hopf algebra, and by the result of Nichols $[\mathrm{N}], \operatorname{gr}(H)$ is twist equivalent to a Nichols Hopf algebra $A:=H_{2^{n}}$.

From this point we will identify $\operatorname{gr}(H)$ and $A$ as quasi-Hopf algebras.

We will now show that $H$ itself is twist equivalent to a Nichols Hopf algebra, completing the proof of the theorem.

Let $I_{r}$ be the radical of $H^{\otimes r}$. Then $I_{r}=\sum_{k=1}^{r} H^{\otimes k-1} \otimes I \otimes H^{\otimes r-k}$.

Let $\Phi$ be the associator of $H$. Then $\Phi=1+\phi$, where $\phi \in I_{3}$. Assume that $\phi \in I_{3}^{m}$, but $\phi \notin I_{3}^{m+1}$. We will show that by twisting we can change $\phi$ so that it will belong to $I_{3}^{m+1}$. Then by a chain of twists we can make sure that $\phi=0$, and by Nichols' result we are done.

Let $\phi^{\prime}$ be the projection of $\phi$ to $I_{3}^{m} / I_{3}^{m+1}=A^{\otimes 3}[m]$ (the $m$ th component in the inherited grading on $A^{\otimes 3}$ ). Then $\phi^{\prime}$ is a Hochschild 3-cocycle of $A^{*}$ with coefficients in the trivial (augmentation) bimodule. But $A^{*}=A=\mathbb{C}\left[\mathbb{Z}_{2}\right] \ltimes \Lambda V$, and

$$
H^{3}(A, \mathbb{C})=H^{3}\left(\mathbb{C}\left[\mathbb{Z}_{2}\right] \ltimes \Lambda V, \mathbb{C}\right)=H^{3}(\Lambda V, \mathbb{C})^{\mathbb{Z}_{2}}=\left(S^{3} V^{*}\right)^{\mathbb{Z}_{2}}=0,
$$

since $\mathbb{Z}_{2}$ acts on $V$ by sign. (For more details on Hochschild cohomology see the book [L].) Thus there exists an element $j^{\prime} \in A^{\otimes 2}[m]$ such that $d j^{\prime}=\phi^{\prime}$. Let $j$ be a lifting of $j^{\prime}$ to $I_{2}^{m}$. Let $J:=1+j$. Let $\Phi^{J}$ be the result of twisting $\Phi$ by $J$, and $\Phi^{J}=1+\phi_{J}$. Then $\phi_{J} \in I_{3}^{m+1}$, as desired. 


\section{Proof of Propositions 3.2,3.3}

5.1. Proof of Proposition 3.2. Let us first show that there exist quasi-Hopf algebras $H_{ \pm}(\infty)$ defined in the same way as $H_{ \pm}(8)$ but without the relation $x^{4}=0$. To show this, it is sufficient to check that $\Phi(\Delta \otimes \mathrm{id}) \Delta(x)=(\mathrm{id} \otimes \Delta) \Delta(x) \Phi$ (the rest of the relations are straightforward). This relation is checked by a simple direct computation.

Now we must show that the ideal generated by $x^{4}$ in the quasi-Hopf algebras $H_{ \pm}(\infty)$ is a quasi-Hopf ideal. To show this, we compute:

$\Delta\left(x^{2}\right)=x^{2} \otimes g+\left[(1+i)\left(p_{+} \otimes p_{+}+p_{-} \otimes p_{+}\right)+(1-i)\left(p_{+} \otimes p_{-}-p_{-} \otimes p_{-}\right)\right](x \otimes x)+g \otimes x^{2}$,

and hence

$$
\Delta\left(x^{4}\right)=x^{4} \otimes 1+1 \otimes x^{4} .
$$

So $x^{4}$ generates a quasi-Hopf ideal, and the quotients $H_{ \pm}(8):=H_{ \pm}(\infty) /\left(x^{4}\right)$ (obviously of dimension 8) are quasi-Hopf algebras. We are done.

5.2. Proof of Proposition 3.3. Let $H_{+-}(\infty)$ be the amalgamated product $H_{+}(8) *_{H(2)} H_{-}(8)$ (i.e. the algebra defined as $H(32)$ but without the relation $x y+i y x=0)$. This is obviously a quasi-Hopf algebra (of infinite dimension). We must show that the principal ideal in $H_{+-}(\infty)$ generated by $z:=x y+i y x$ is a quasi-Hopf ideal. This follows from the easily established relation $\Delta(z)=$ $z \otimes 1+g \otimes z$.

Thus, $H(32):=H_{+-}(\infty) /(z)$ is a quasi-Hopf algebra. It is easy to show that the elements $g^{j} x^{k} y^{l}, j=0,1, k, l=0,1,2,3$, form a basis in $H(32)$. Thus, $H(32)$ is 32 -dimensional.

\section{Proof of Theorem 3.4}

Let $p_{+}, p_{-}$be the primitive idempotents of $H[0]$, and let $g:=p_{+}-p_{-}$. Let $\Phi_{0}$ be the associator of $\operatorname{gr}(H)$. By using a twist, we may assume that $\Phi_{0}=1-2 p_{-} \otimes p_{-} \otimes p_{-}$(the only, up to equivalence, nontrivial associator for $\left.\mathbb{C}\left[\mathbb{Z}_{2}\right]\right)$. Let $\chi$ be the nontrivial character of $H[0]$.

Let $x$ be an element of $H[1]$. By Theorem 2.17 in [EO], $\operatorname{Ext}^{1}(\varepsilon, \varepsilon)=\operatorname{Ext}^{1}(\chi, \chi)$ $=0$, thus by subsection 2.5 ,

$$
g x g^{-1}=-x .
$$

For $z \in H$ define

$$
\xi(z):=(\chi \otimes \mathrm{id})(\Delta(z)) \text { and } \eta(z):=(\mathrm{id} \otimes \chi)(\Delta(z)) .
$$

Then $\xi(g)=\eta(g)=-g$.

For $x \in H[1]$ we have that $\Delta(x) \in H[0] \otimes H[1] \oplus H[1] \otimes H[0]$. Writing $\Delta(x)=a \otimes p_{+}+p_{+} \otimes b+c \otimes p_{-}+p_{-} \otimes d$ and applying $\chi^{i} \otimes \chi^{j}, i, j=0,1$, yields

$$
\Delta(x)=x \otimes p_{+}+p_{+} \otimes x+\eta(x) \otimes p_{-}+p_{-} \otimes \xi(x) .
$$

By the quasi-coassociativity axiom, we have $\Phi_{0}(\Delta \otimes \mathrm{id}) \Delta(x)=(\mathrm{id} \otimes \Delta) \Delta(x) \Phi_{0}$. 
Lemma 6.1. The equation $\Phi_{0}(\Delta \otimes i d) \Delta(x)=(i d \otimes \Delta) \Delta(x) \Phi_{0}$ is equivalent to the relations

$$
\xi^{2}(x)=-x, \eta^{2}(x)=-x, \text { and } \xi \eta(x)=-\eta \xi(x) .
$$

Proof. For degree reasons the coassociativity equation is equivalent to the system of three equations obtained by applying $\chi \otimes \chi \otimes \mathrm{id}, \chi \otimes \mathrm{id} \otimes \chi$, and id $\otimes \chi \otimes \chi$. Since

$$
(\chi \otimes \chi \otimes \mathrm{id})\left(\Phi_{0}\right)=(\chi \otimes \mathrm{id} \otimes \chi)\left(\Phi_{0}\right)=(\mathrm{id} \otimes \chi \otimes \chi)\left(\Phi_{0}\right)=g,
$$

application of $\chi \otimes \mathrm{id} \otimes \chi$ gives

$$
\begin{aligned}
& \xi \eta(x)=\xi((\mathrm{id} \otimes \chi)(\Delta(x))) \\
& \quad=(\chi \otimes \mathrm{id} \otimes \chi)((\mathrm{id} \otimes \Delta) \Delta(x)) \\
& \quad=(\chi \otimes \mathrm{id} \otimes \chi)\left(\Phi_{0}(\Delta \otimes \mathrm{id}) \Delta(x) \Phi_{0}^{-1}\right) \\
& \quad=g(\chi \otimes \mathrm{id} \otimes \chi)((\Delta \otimes \mathrm{id}) \Delta(x)) g^{-1} \\
& \quad=g \eta \xi(x) g^{-1},
\end{aligned}
$$

which is equivalent to $\xi \eta(x)=-\eta \xi(x)$.

Also, application of $\chi \otimes \chi \otimes$ id yields

$$
\begin{aligned}
& \xi^{2}(x)=\xi((\chi \otimes \mathrm{id})(\Delta(x))) \\
& \quad=(\chi \otimes \chi \otimes \mathrm{id})((\mathrm{id} \otimes \Delta) \Delta(x)) \\
& \quad=(\chi \otimes \chi \otimes \mathrm{id})\left(\Phi_{0}(\Delta \otimes \mathrm{id}) \Delta(x) \Phi_{0}^{-1}\right)=g x g^{-1}=-x
\end{aligned}
$$

and similarly application of id $\otimes \chi \otimes \chi$ yields $\eta^{2}(x)=-x$.

Let $L_{g}$ be the operator of left multiplication by $g$ in $H[1]$. Then $L_{g}^{2}=$ id, and $L_{g} \xi=-\xi L_{g}, L_{g} \eta=-\eta L_{g}$. Thus, using Lemma 6.1, we see that the operators $\xi, \eta, L_{g}$ define an action on $H[1]$ of the Clifford algebra $C l_{3}$ of a 3 -dimensional inner product space.

The following lemma is standard.

Lemma 6.2. The algebra $\mathrm{Cl}_{3}$ is semisimple and has two irreducible representations $W_{ \pm}$, which are both 2-dimensional. They are spanned by elements $x$ and gx with $\eta(x)=i x, \xi(x)= \pm g x$.

(Here we abuse the notation by writing $g$ instead of $L_{g}$.)

Lemma 6.2 implies that as a $C l_{3}$-module, $H[1]=n W_{+} \oplus m W_{-}$.

Let $x \in W_{ \pm} \subseteq H[1]$ be an eigenvector of $\eta$ with eigenvalue $i$. Then we have

$$
\begin{aligned}
& \Delta(x)=x \otimes\left(p_{+} \pm i p_{-}\right)+p_{+} \otimes x+p_{-} \otimes g x \\
& \quad=\quad x \otimes\left(p_{+} \pm i p_{-}\right)+1 \otimes p_{+} x+g \otimes p_{-} x .
\end{aligned}
$$

Together with Lemma 2.2 (ii), this implies the following proposition:

Proposition 6.3. The quasi-Hopf algebra $g r(H)$ is generated by elements $g$ of degree 0 , and $x_{1}, \ldots, x_{n}, y_{1}, \ldots, y_{m}$ of degree 1 , which satisfy the relations $g x_{j}=$ 
$-x_{j} g, g y_{j}=-y_{j} g, g^{2}=1$ (and possibly other relations). The coproduct of $g r(H)$ is defined by the formulas

$$
\begin{gathered}
\Delta(g)=g \otimes g, \\
\Delta\left(x_{j}\right)=x_{j} \otimes\left(p_{+}+i p_{-}\right)+1 \otimes p_{+} x_{j}+g \otimes p_{-} x_{j}, \\
\Delta\left(y_{j}\right)=y_{j} \otimes\left(p_{+}-i p_{-}\right)+1 \otimes p_{+} y_{j}+g \otimes p_{-} y_{j} .
\end{gathered}
$$

Lemma 6.4. In $\operatorname{gr}(H)$, we have $x_{j}^{4}=y_{l}^{4}=0$ for all $j, l$.

Proof. It suffices to show that $x_{j}^{4}=0$, the case of $y_{l}$ is obtained by changing $i$ to $-i$. Let $x=x_{j}$. Using Proposition 6.3 we find that

$\Delta\left(x^{2}\right)=x^{2} \otimes g+\left[(1+i)\left(p_{+} \otimes p_{+}+p_{-} \otimes p_{+}\right)+(1-i)\left(p_{+} \otimes p_{-}-p_{-} \otimes p_{-}\right)\right](x \otimes x)+g \otimes x^{2}$,

hence

$$
\Delta\left(x^{4}\right)=x^{4} \otimes 1+1 \otimes x^{4} .
$$

But a finite dimensional quasi-Hopf algebra cannot contain nonzero primitive elements (this is proved as in the Hopf case). Thus, $x^{4}=0$.

Lemma 6.5. The numbers $m$ and $n$ are either 0 or 1 .

Proof. Clearly, it suffices to show that $n=0$ or 1 . Assume the contrary, i.e. that $n \geq 2$.

Introduce the element $z:=g\left(x_{2} x_{1}-i x_{1} x_{2}\right)$. Using Proposition 6.3 it is checked directly that

$$
\Delta(z)=z \otimes 1+1 \otimes z+2\left(g x_{2} \otimes p_{+} x_{1}+i x_{2} \otimes p_{-} x_{1}\right) .
$$

Let $N$ be the smallest integer such that $z^{N}=0$. It exists since $\operatorname{gr}(H)$ is finite dimensional. Taking the coproduct of this equation and looking at the terms of bidegree $(2 N-1,1)$, we find

$$
\sum_{k=1}^{N} z^{k-1} x_{2} z^{N-k}=0 .
$$

Let us now apply the coproduct to this equality, and look at the terms of bidegree $(2 N-2,1)$ which have a factor $x_{2}$ in the second component. This yields $N z^{N-1}=$ 0 , which is a contradiction with the minimality of $N$.

Lemma 6.6. (i) If $m=n=0, \operatorname{gr}(H)=H(2)$.

(ii) If $n=1, m=0$ then $\operatorname{gr}(H)=H_{+}(8)$.

(iii) If $n=0, m=1$ then $\operatorname{gr}(H)=H_{-}(8)$.

(iv) If $n=1, m=1$ then $\operatorname{gr}(H)=H(32)$.

Proof. Statements (i)-(iii) of the lemma follow from the arguments above, since it is easy to check that the algebras $H_{ \pm}(8)$ do not have nontrivial graded quasiHopf ideals which do not intersect with $H[1]$. It remains to prove statement (iv).

Assume $m=n=1$, and set $x:=x_{1}, y:=y_{1}$. Consider the element $z:=$ $x y+i y x$. A direct computation shows that

$$
\Delta(z)=z \otimes 1+g \otimes z .
$$


Since $g z=z g$ and $\operatorname{gr}(H)$ is finite dimensional, we must have $z=0$ (this is shown exactly in the same way as one shows that nonzero primitive elements do not exist). Thus, $\operatorname{gr}(H)$ is a quotient of $H(32)$. But it is easy to check directly that $H(32)$ does not have nontrivial graded quasi-Hopf ideals which do not intersect with $H[1]$. We are done.

Lemma 6.7. $H_{+}(8)$ is not twist equivalent to $H_{-}(8)$.

Proof. Suppose they are. Then there exists a twist $J$ for $H_{-}(8)$, and an isomorphism of algebras $f: H_{+}(8) \rightarrow H_{-}(8)^{J}$. Such an isomorphism obviously preserves filtration by powers of the radical, so we can take its degree zero part. Thus we can assume, without loss of generality, that $f$ preserves the grading and $J$ is of degree zero. Then $f$ is the identity on the degree zero part, and $\Phi^{J}=\Phi$. So $J$ is a twist of $\mathbb{C}\left[\mathbb{Z}_{2}\right]$, hence a coboundary, and thus we may assume that $J=1 \otimes 1$ (by changing $f$ ). But then $f$ cannot exist, since $S^{2}(a)=i a$ on $H_{+}(8)[1]$ and $S^{2}(a)=-i a$ on $H_{-}(8)[1]$.

This completes the proof of Theorem 3.4.

\section{Relation to pointed Hopf algebras}

Let $H=\oplus_{k \geq 0} H[k]$ be a graded quasi-Hopf algebra with radical of codimension 2 and nontrivial associator on 1-dimensional representations.

The main result of this section is that it is actually possible to embed $H$ into a twice bigger quasi-Hopf algebra $\tilde{H}$, which is twist equivalent to a basic Hopf algebra $H^{\prime}$ with $H^{\prime} / \operatorname{Rad}\left(H^{\prime}\right)=\mathbb{C}\left[\mathbb{Z}_{4}\right]$. This fact should have generalizations to the case of general graded basic quasi-Hopf algebras, which may facilitate applications to the quasi-Hopf case of known deep results about pointed Hopf algebras.

To construct $\tilde{H}$, let us adjoin a new element $a$ to $H$ which is grouplike, $a^{2}=g$, and $a z a^{-1}=i^{k} z$ for $z \in H[k]$. Then the algebra $\tilde{H}$ generated by $a$ and $H$ (of twice bigger dimension than that of $H$ ) is a quasi-Hopf algebra graded by nonnegative integers, with $\tilde{H}[0]=\mathbb{C}\left[\mathbb{Z}_{4}\right]$.

Proposition 7.1. $\tilde{H}$ is twist equivalent to a Hopf algebra.

Proof. We claim that the image of the associator $\Phi_{0}$ in $\tilde{H}[0]^{\otimes 3}$ is homologically trivial. This is because the natural map $f^{\prime}: H^{3}\left(\mathbb{Z}_{2}, \mathbb{C}^{*}\right) \rightarrow H^{3}\left(\mathbb{Z}_{4}, \mathbb{C}^{*}\right)$ induced by the projection $f: \mathbb{Z}_{4} \rightarrow \mathbb{Z}_{2}$ is zero. Indeed, for a cyclic group $G$ one has $H^{3}\left(G, \mathbb{C}^{*}\right)=G^{*} \otimes G^{*}$ (functorially in $G$ ). Thus for any morphism of cyclic groups $f: G_{1} \rightarrow G_{2}$, the induced map of third cohomology groups is $f^{\prime}=f^{*} \otimes f^{*}$. In our case $f: \mathbb{Z}_{4} \rightarrow \mathbb{Z}_{2}$, so $f^{*}: \mathbb{Z}_{2} \rightarrow \mathbb{Z}_{4}$ is given by $f^{*}(1)=2$. Hence, $\left(f^{*} \otimes f^{*}\right)(1 \otimes 1)=2 \otimes 2=1 \otimes 4=0$, as desired.

Thus, by a twist in $\tilde{H}[0]^{\otimes 2}$, we can kill $\Phi_{0}$. So $\tilde{H}$ is twist equivalent to a graded pointed Hopf algebra $A$ generated in degree 1 with $A / \operatorname{Rad}(A)=\mathbb{C}\left[\mathbb{Z}_{4}\right]$. 
Example 7.2. Let $A_{ \pm}(16)$ (for each choice of sign) be the Hopf algebra of dimension 16 generated by $a, x$ with relations $a^{4}=1, a x a^{-1}=i x, x^{4}=0$, such that $a$ is grouplike and $\Delta(x)=1 \otimes x+x \otimes a^{\mp 1}$.

Let $A(64)$ be the Hopf algebra of dimension 64 generated by $a, x, y$ with relations $a^{4}=1, a x a^{-1}=i x, a y a^{-1}=i y, x^{4}=0, y^{4}=0, x y+i y x=0$, such that $a$ is grouplike and $\Delta(x)=1 \otimes x+x \otimes a^{-1}, \Delta(y)=1 \otimes y+y \otimes a$.

In other words, $A_{ \pm}(16)=u_{q}\left(\mathfrak{b}_{+}\right)$, where $\mathfrak{b}_{+}$is the Borel subalgebra in $\mathfrak{s} l_{2}$, and $q=\exp ( \pm \pi i / 4)$, while $A(64)=\operatorname{gr}\left(u_{q}\left(\mathfrak{s} l_{2}\right)^{*}\right)$, for the same $q$.

Proposition 7.3. (i) $\widetilde{H_{ \pm}(8)}$ is twist equivalent to $A_{ \pm}(16)$.

(ii) $\widetilde{H(32)}$ is twist equivalent to $A(64)$.

Proof. (i) Consider the Hopf algebra $A$ obtained by twisting away the associator in $\widehat{H_{+}(8)}$. Then $A=\bigoplus_{k \geq 0} A[k]$ is a 16 -dimensional graded basic (and pointed) Hopf algebra generated in degree 1. Grouplike elements of this Hopf algebra form a group $\mathbb{Z}_{4}$, and $A[1]$ is a free rank 1 module over $A[0]$ under left multiplication. So $A=A_{+}(16)$ or $A=A_{-}(16)$. To decide the sign, assume that $J$ is a pseudotwist for $A_{s}(16)$, and $f: \widetilde{H_{+}(8)} \rightarrow A_{s}(16)^{J}$ is an isomorphism (where $s$ is a choice of sign). Since $f$ preserves the filtration by powers of the radical, we may assume (by taking the degree 0 part) that $f$ preserves the grading and $J$ has degree 0 . Then $\Phi=(1 \otimes 1 \otimes 1)^{J}$. This implies that if $Q:=\sum d_{j} S\left(e_{j}\right)$, where $J^{-1}=\sum d_{j} \otimes e_{j}$, then $Q S(Q)^{-1}=a^{2}$. Hence the squares of the antipodes in $\widetilde{H_{+}(8)}$ and $A_{s}(16)$ differ by conjugation by $a^{2}$, i.e. by a sign on the degree 1 component. For $\widetilde{H_{+}(8)}$, the eigenvalue of $S^{2}$ in degree 1 is $i$. Thus $s=+$.

(ii) Consider the Hopf algebra $A$ obtained by twisting away the associator in $\widehat{H(32)}$. This is a 64-dimensional graded basic (and pointed) Hopf algebra, which is a quotient of the amalgamated product of $A_{+}(16)$ (generated by $a, x$ ) and $A_{-}(16)$ (generated by $\left.a, y\right)$. It is easy to see that $x y+i y x$ is a primitive element, so it must be zero. Thus, $A=A(64)$, which is the quotient of this amalgamated product by the relation $x y+i y x=0$.

\section{Acknowledgments}

We thank V. Ostrik for suggesting to generalize the result of Nichols to the quasi-Hopf case.

The first author was partially supported by the NSF grant DMS-9988796. The second author's research was supported by Technion V.P.R. Fund - Dent Charitable Trust - Non Military Research Fund, and by The Israel Science Foundation (grant No. 70/02-1). He is also grateful to MIT for its warm hospitality. Both authors were partially supported by the BSF grant No. 2002040. 


\section{References}

[AS] N. Andruskiewitsch and H-J. Schneider, Pointed Hopf algebras. New directions in Hopf algebras, 1-68, Math. Sci. Res. Inst. Publ., 43, Cambridge Univ. Press, Cambridge, 2002.

[CE] D. Calaque and P. Etingof, Lectures on tensor categories, math.QA/0401246.

[D] V. Drinfeld, Quasi-Hopf algebras. (Russian) Algebra i Analiz 1 (1989), 114-148; translation in Leningrad Math. J. 1 (1990), 1419-1457.

[E] P. Etingof, On Vafa's theorem for tensor categories, Math. Res. Lett. 9 (2002), 651-657.

[ENO] P. Etingof, D. Nikshych and V. Ostrik, On fusion categories, Annals of Mathematics, to appear, math.QA/0203060.

[EO] P. Etingof and V. Ostrik, Finite tensor categories, submitted, math.QA/0301027.

[L] J-L. Loday, Cyclic homology, Grundlehren der mathematischen Wissenschaften in Einzeldarstellungen $\mathbf{3 0 1}$ (1992).

[N] W. Nichols, Bialgebras of type one, Comm. Algebra 6 (1978), 1521-1552.

[S] M. Sweedler, Hopf Algebras, Benjamin Press, 1968.

[TY] D. Tambara and S. Yamagami, Tensor categories with fusion rules of self-duality for finite abelian groups, J. Algebra 209 (1998), 692-707.

Department of Mathematics, Massachusetts Institute of Technology, Cambridge, MA 02139, USA

E-mail address: etingof@math.mit.edu

Department of Mathematics, Technion-Israel Institute of Technology, Haifa 32000, ISRAEL

E-mail address: gelaki@math.technion.ac.il 